\title{
Fine-grained energy profiling for deep convolutional neural networks on the Jetson TX1
}

DOI:

10.1109/IISWC.2017.8167764

\section{Document Version}

Accepted author manuscript

Link to publication record in Manchester Research Explorer

\section{Citation for published version (APA):}

Rodrigues, C., Riley, G., \& Luján, M. (2017). Fine-grained energy profiling for deep convolutional neural networks on the Jetson TX1. In IEEE International Symposium on Workload Characterization (IISWC), 2017 (pp. 114-115) https://doi.org/10.1109/IISWC.2017.8167764

\section{Published in:}

IEEE International Symposium on Workload Characterization (IISWC), 2017

\section{Citing this paper}

Please note that where the full-text provided on Manchester Research Explorer is the Author Accepted Manuscript or Proof version this may differ from the final Published version. If citing, it is advised that you check and use the publisher's definitive version.

\section{General rights}

Copyright and moral rights for the publications made accessible in the Research Explorer are retained by the authors and/or other copyright owners and it is a condition of accessing publications that users recognise and abide by the legal requirements associated with these rights.

\section{Takedown policy}

If you believe that this document breaches copyright please refer to the University of Manchester's Takedown Procedures [http://man.ac.uk/04Y6Bo] or contact uml.scholarlycommunications@manchester.ac.uk providing relevant details, so we can investigate your claim.

\section{OPEN ACCESS}




\section{Fine-Grained Energy Profiling for Deep Convolutional Neural Networks on the Jetson TX1}

\author{
Crefeda Faviola Rodrigues \\ School of Computer Science \\ The University of Manchester \\ Manchester, UK
}

\author{
Graham Riley \\ School of Computer Science \\ The University of Manchester \\ Manchester, UK
}

\author{
Mikel Luján \\ School of Computer Science \\ The University of Manchester \\ Manchester,UK
}

Email: crefeda.rodrigues@manchester.ac.uk Email: graham.riley@manchester.ac.uk Email: mikel.lujan@manchester.ac.uk

\begin{abstract}
Energy-use is a key concern when migrating current deep learning applications onto low power heterogeneous devices such as a mobile device. This is because deep neural networks are typically designed and trained on high-end GPUs or servers and require additional processing steps to deploy them on low power devices. Such steps include the use of compression techniques to scale down the network size or the provision of efficient device-specific software implementations. Migration is further aggravated by the lack of tools and the inability to measure power and performance accurately and consistently across devices. We present a novel evaluation framework for measuring energy and performance for deep neural networks using ARMs Streamline Performance Analyser integrated with standard deep learning frameworks such as Caffe and CuDNNv5. We apply the framework to study the execution behaviour of SqueezeNet on the Maxwell GPU of the NVidia Jetson TX1, on an image classification task (also known as inference) and demonstrate the ability to measure energy of specific layers of the neural network.
\end{abstract}

\section{INTRODUCTION}

Recent fast-paced developments of Deep neural networks in various application domains such as computer vision and natural language processing, gaming and others has resulted in the emergence of innovative applications being developed for mobile and embedded systems [1]. These systems pose constraints on the demand for computational resources and power which requires rethinking in several aspects: algorithm design, software implementation and hardware design. Deep neural networks such as Convolutional Neural Networks (hereafter referred to as ConvNets) [2] are commonly employed in vision-based applications. To enable deep learning on these "edge devices", optimization efforts are spreads across all levels: compact model designs [3]-[5], compression and pruning [6], [7], reduced precision [8], scheduling strategies [9], device-specific software implementations [10], [11] and application-specific hardware [2], [12].

Currently, accuracy ${ }^{1}$ is the most common metric to evaluate a neural network model while performance or time is an important metric to characterize deep learning workloads (for example, Fathom workloads [1] and ConvNet implementation benchmarks on desktop systems [13]). Owing to the difficulty in measuring energy, only a few studies exists that actually

\footnotetext{
${ }^{1}$ top- 1 or top- 5 accuracy for image classification task
}

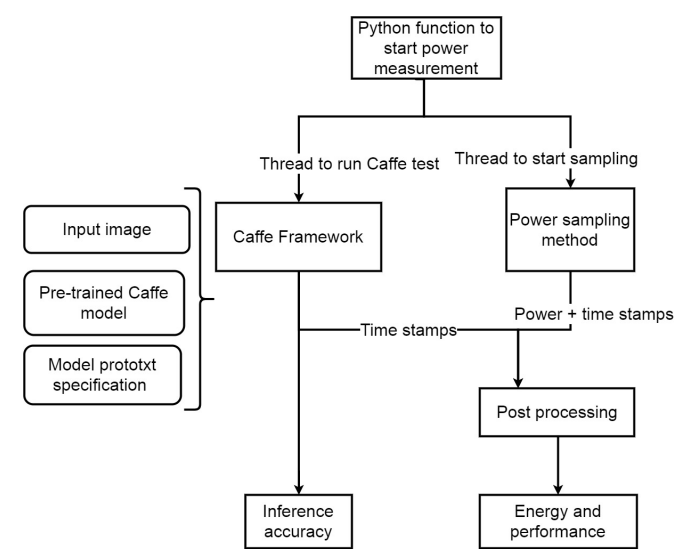

Fig. 1. Overview of the evaluation framework

measure energy consumption of machine learning algorithms such as Decision Trees [14] and coarse-grained energy measurements for inferences on a mobile device [15]. Our work focuses on enabling researchers to evaluate existing and future ConvNet models on the metric of energy-use in addition to accuracy and performance.

We propose the development of an evaluation framework, as shown in Figure 1, to support coarse and fine-grained energy profiling to provide visibility into the energy consumption during the inference phase of deep neural networks on a mobile device. Our methodology focusses on power measurements made using the on-board power monitoring sensor TIINA3221x [16] available on the Jetson TX1. The TX1 runs with Linux kernel (3.10.96) and Ubuntu 16.04. To measure energy at a fine-grained level we integrate vendor-specific tools such as ARM Streamline Performance Analyzer [17] to the Caffe deep learning framework [18].

\section{Evaluation Framework And Results}

The main component of the Evaluation Framework is its power sampling method that reads the power samples from the power monitor unit through the sysfs. The power monitor provides System-level power at VDD_IN , CPU-level power at VDD_CPU and GPU-level power at VDD_GPU. With the help of ARM Streamline, annotation markers were inserted into the code to mark the beginning of each layer in the neural 


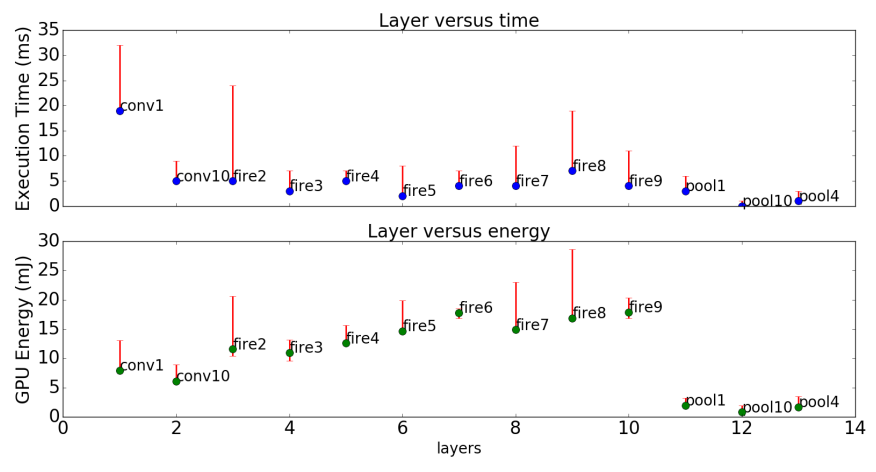

Fig. 2. Per-layer performance and energy profile of SqueezeNet

network. These time stamps were then correlated to the time stamps of the power values, sampled at intervals of $1 \mathrm{~ms}$. Our Caffe (version 1.0.0-rc3) was compiled for the GPU with Cuda (8.0) and CuDNN (5.1.5) and CPU with OpenBLAS (libopenblas_cortexa57p-r.0.2.20.dev.a) with a max num-thread of 4 . The application selected was an inference using SqueezeNet model [3] on a single RGB image ( $224 \times 224$ pixels) taken from ImageNet dataset [19].

From Figure 2, we observe similar trends in performance and energy measures for certain layers in SqueezeNet (for example, conv and pooling layers). Note that the conv layers and the fire layers are essentially convolution operations. We observe an increase in energy consumption trend after each fire module (for example, fire $3<$ fire $4<$ fire5). We hypothesize some of the factors that could explain this behaviour after examining the GPU traces from nvprof. We observe that for a convolution, there exist at least three $C u D N N$ implementations: gemm, winograd and implicit_convolve_sgemm, the choice of which is based on the kernel size of each convolution. The fire modules are composed of one or more of these implementations since they are composed of $(1 \times 1$ and $3 \times 3$ ) kernels sizes. In contrast, conv layers that are closest to the input are composed of larger kernel sizes $(7 \times 7$ or $5 \times 5$ ) rely on gemm implementations. Understanding the implications on performance and energy of choosing one type of implementation over the other, could help provide a better understanding of which choices lead to better energyefficiency. Therefore, we demonstrate that measuring energy in conjunction with performance, should offer better insights to enable better application-specific optimization strategies and guide the design of energy efficient ConvNets.

\section{Conclusion And Future Scope}

Energy optimization efforts have emerged to enable deep learning applications to execute efficiently on current mobile systems. Understanding the energy-use at specific phases (such as layer-wise energy consumption) of the application in terms of computation and data movement backed-up with actual energy measurements should help researchers develop better models of energy consumption and guide the development of application-specific optimizations for deep neural networks on mobile devices. For example, understanding the impact of different implementation choices on the energy-efficiency for a convolution operation.

The opportunities for future work include characterizing these algorithms and providing a model for energy consumption to understand which design choices (layer types, filter sizes) in the model architecture leads to energy-savings.

\section{REFERENCES}

[1] R. Adolf, S. Rama, B. Reagen, G.-Y. Wei, and D. Brooks, "Fathom: reference workloads for modern deep learning methods," in Workload Characterization (IISWC), 2016 IEEE International Symposium on. IEEE, 2016, pp. 1-10.

[2] V. Sze, Y.-H. Chen, T.-J. Yang, and J. Emer, "Efficient processing of deep neural networks: A tutorial and survey," arXiv preprint arXiv:1703.09039, 2017.

[3] F. N. Iandola, M. W. Moskewicz, K. Ashraf, S. Han, W. J. Dally, and K. Keutzer, "Squeezenet: Alexnet-level accuracy with 50x fewer parameters andi $1 \mathrm{mb}$ model size," arXiv preprint arXiv:1602.07360, 2016.

[4] C. Szegedy, W. Liu, Y. Jia, P. Sermanet, S. Reed, D. Anguelov, D. Erhan, V. Vanhoucke, and A. Rabinovich, "Going deeper with convolutions," in Proceedings of the IEEE Conference on Computer Vision and Pattern Recognition, 2015, pp. 1-9.

[5] A. G. Howard, M. Zhu, B. Chen, D. Kalenichenko, W. Wang, T. Weyand, M. Andreetto, and H. Adam, "Mobilenets: Efficient convolutional neural networks for mobile vision applications," arXiv preprint arXiv:1704.04861, 2017.

[6] S. Han, H. Mao, and W. J. Dally, "Deep compression: Compressing deep neural networks with pruning, trained quantization and huffman coding," arXiv preprint arXiv:1510.00149, 2015.

[7] T.-J. Yang, Y.-H. Chen, and V. Sze, "Designing energy-efficient convolutional neural networks using energy-aware pruning," arXiv preprint arXiv:1611.05128, 2016.

[8] M. Courbariaux, Y. Bengio, and J.-P. David, "Training deep neural networks with low precision multiplications," arXiv preprint arXiv:1412.7024, 2014.

[9] N. D. Lane, S. Bhattacharya, P. Georgiev, C. Forlivesi, L. Jiao, L. Qendro, and F. Kawsar, "Deepx: A software accelerator for low-power deep learning inference on mobile devices," in 2016 15th ACM/IEEE International Conference on Information Processing in Sensor Networks (IPSN). IEEE, 2016, pp. 1-12.

[10] S. Chetlur, C. Woolley, P. Vandermersch, J. Cohen, J. Tran, B. Catanzaro, and E. Shelhamer, "cudnn: Efficient primitives for deep learning," arXiv preprint arXiv:1410.0759, 2014.

[11] 2017. [Online]. Available: https://developer.arm.com/technologies/ compute-library

[12] 2016. [Online]. Available: https://cloudplatform.googleblog.com/2016/ 05/Google-supercharges-machine-learning-tasks-with-custom-chip. html

[13] 2016. [Online]. Available: https://github.com/soumith/ convnet-benchmarks

[14] E. Garcia-Martin, N. Lavesson, and H. Grahn, "Identification of energy hotspots: A case study of the very fast decision tree," in International Conference on Green, Pervasive, and Cloud Computing. Springer, 2017, pp. 267-281.

[15] N. D. Lane, S. Bhattacharya, P. Georgiev, C. Forlivesi, and F. Kawsar, "An early resource characterization of deep learning on wearables, smartphones and internet-of-things devices," in Proceedings of the 2015 International Workshop on Internet of Things towards Applications. ACM, 2015, pp. 7-12.

[16] 2017. [Online]. Available: http://www.ti.com/product/INA3221/ datasheet

[17] 2016. [Online]. Available: https://developer.arm.com/products/ software-development-tools/ds-5-development-studio/streamline

[18] Y. Jia, E. Shelhamer, J. Donahue, S. Karayev, J. Long, R. Girshick, S. Guadarrama, and T. Darrell, "Caffe: Convolutional architecture for fast feature embedding," in Proceedings of the ACM International Conference on Multimedia. ACM, 2014, pp. 675-678.

[19] A. Berg, J. Deng, and L. Fei-Fei, "Large scale visual recognition challenge (ilsvrc), 2010," URL http://www. image-net. org/challenges/LSVRC, 2010. 